\title{
Câncer da mama na cadela
}

\section{Mammary cancer in female dogs}

Vítor Brasil Medeiros

Review performed in Natal, Rio Grande do Norte, Brazil.

Financial support: None.

Conflict of interest: None.

Correspondence address: Ave. Miguel Alcides Araújo 1889, Natal, RN, Brazil. E-mail: vitor_brasilm@hotmail.com

Submitted: september 03, 2017. Accepted, after review: september 25, 2017.

\begin{abstract}
Purpose: This paper aimed to do an introduction to female canine mammary cancer, including differential characteristics, frequency and hormone dependence, diagnosis and treatment. Methods: A review of the literature in Pubmed database was performed using the key words canine mammary tumors, pathogenesis, staging, diagnosis, treatment. Results: Mammary tumours were discussed regarding hormones and mammary cancer, Tumor types, histopathologic classification, and biologic behavior, Clinical signs, physical exam, diagnostic and treatment. Based on the clinical information available in veterinary medicine, the drugs that are effective in breast cancer are cyclophosphamide, 5-fluorouracil, and doxorubicin, which have an important role in the treatment of malignant mammary gland tumors in dogs. The treatment of canine mammary gland tumors must be based on the individual oncologist's understanding of tumor biology, experience, and interpretation of the available studies. Finally, the article deals briefly with the development of new treatments and uptodate about many aspects in the management of mammary cancer in female dogs.
\end{abstract}

KEYWORDS: Canine mammary cancer. Pathogenesis. Progesterone. Hormones. Treatment. 


\section{RESUMO}

Objetivo: Este artigo teve como objetivo fazer uma revisão sobre o câncer de mama canino feminino, incluindo características diferenciais, frequência e dependência hormonal, diagnóstico e tratamento. Métodos: uma revisão da literatura na base de dados Pubmed foi realizada usando as palavras-chave tumores mamários caninos, patogênese, estadiamento, diagnóstico, tratamento. Resultados: Foram discutidos aspectos relevantes da influência dos hormônios no câncer de mama em cadelas, tipos de tumores, classificação histopatológica e comportamento biológico, sinais clínicos, exame físico, diagnóstico e tratamento. Com base na informação clínica disponível em medicina veterinária, os medicamentos quimioterápicos ciclofosfamida, 5fluorouracilo e doxorrubicina, podem desempenhar um papel importante no tratamento de tumores malignos das glândulas mamárias em cães. O tratamento deve basear-se na compreensão do oncologista individual sobre biologia do tumor, experiência e interpretação dos estudos disponíveis. Finalmente, o artigo aborda o uso de novos tratamentos e atualiza sobre muitos aspectos no manejo do câncer de mama em cães do sexo feminino.

DESCRITORES: Câncer de mama canino. Patogênese. Progesterona. Hormônios. Tratamento.

\section{INTRODUÇÃO}

Os tumores mamários são as neoplasias mais comuns em cadelas. Cerca de 50\% destes tumores são malignos e um terço pode formar metástases à distância que ameaçam a vida. A organização celular hierárquica da glândula mamária tornou-se cada vez mais clara e está ligada à heterogeneidade do epitélio mamário, com uma camada externa e interna de células. Ambas as camadas possuem diferentes células epiteliais com características diferenciadas. Na camada basal externa de células mioepiteliais contráteis, as células tronco mamárias, enquanto que na camada de células luminais epiteliais ductais, as alveolares, e as células progenitoras ${ }^{2,3}$.

Os tumores das glândulas mamárias constituem $13,4 \%$ de todos os tumores em cães e $41,7 \%$ de todos os tumores em fêmeas. A incidência de tumores das glândulas mamárias caninas nos Estados Unidos, no entanto, foi reduzida significativamente desde que foi instituída a prática comum de realizar ovariohisterectomia em uma idade precoce. Os tumores da glândula mamária são muito mais comuns em muitos países europeus em que a ovário-histerectomia não é 
Câncer da mama na cadela

Medeiros VB

rotineiramente realizada. Os dados recentes do The Norwegian Canine Cancer Registry relataram uma incidência de tumores malignos das glândulas mamárias de 53,3\% em cães do sexo feminino de qualquer raça ${ }^{4}$. Esse número parece alto, mas pode ser influenciado pelo uso comum de progestágenos para prevenir estrus nesta população em particular. Um estudo prévio do mesmo grupo encontrou um risco aumentado, com um odds ratio de 2,32, do desenvolvimento do tumor de glândula mamária em cães tratados com progestágenos ${ }^{5}$. Os estrogênios e as progesteronas desempenham um papel importante no desenvolvimento normal das glândulas mamárias, mas estes hormônios também são implicados no desenvolvimento de tumores ${ }^{6}$. Os estrogênios são promotores de células jovens, além de regular a transcrição de vários protooncogenes nucleares ${ }^{7}$.

O principal problema no tratamento do câncer de mama é a recorrência do crescimento tumoral e metástases. Em ambos os casos, as células-tronco do câncer têm uma função importante. O paradigma atual é que uma célula tronco comum dá origem a células progenitoras que são intermediárias nas linhagens de células mioepiteliais e epiteliais ou lobulares ${ }^{8}$. Nos cães, um subconjunto de carcinomas mamários apresenta-se como carcinomas simples que podem ser derivados de células progenitoras epiteliais mutantes e são comparáveis à forma mais comum de câncer de mama humano, os carcinomas ductais. No entanto, mais frequentemente do que em seres humanos, os cães também podem apresentar carcinomas complexos que contêm várias vias de diferenciação dentro de uma única glândula mamária afetada. Estes tumores podem ser derivados de mutações na forma mais básica e precoce das células tronco. A existência de células tronco e a presença de várias formas progenitoras podem, em parte, explicar a heterogeneidade dos carcinomas mamários ${ }^{9}$.

\section{Hormônios e câncer de mama}

O papel central da sinalização de progesterona no desenvolvimento do câncer de mama ganhou interesse renovado após o grande estudo de reposição hormonal realizado em $2003^{10}$. Sabe-se que os esteroides ovarianos desempenham um papel importante na carcinogênese da glândula mamária ${ }^{11}$. O papel carcinogênico da progesterona tem sido subestimado há muito tempo ${ }^{12}$ devido ao uso mais 
Câncer da mama na cadela

Medeiros VB

generalizado de progestinas sintéticas como inibidores do crescimento tumoral ${ }^{13}$. Em relação à fisiologia reprodutiva, a progesterona pode ter um papel protetor ou pode ser um fator de risco para o câncer de mama. O papel protetor da progesterona é visto em mulheres com gravidez e aleitamento precoce a termo. Os fatores de risco associados ao aumento da exposição à progesterona são os intervalos prolongados representados pela menarca e idade do primeiro parto, idade mais avançada na menopausa, menarca precoce, e menopausa $\operatorname{tardia}^{14}$.

O hormônio do crescimento pituitário (HC) também é necessário para o desenvolvimento da glândula mamária. A deficiência de HC prejudica o desenvolvimento mamário em ratos e camundongos. O HC influencia o desenvolvimento alveolar e ductal da mama ${ }^{15}$. As mulheres com hipersecreção de HC (acromegalia) e indivíduos com maior altura possuem maior incidência de câncer de mama ${ }^{18}$. Uma exposição prolongada aos hormônios esteroides é o fator de risco mais bem estabelecido para o câncer de mama humano ${ }^{16}$, o que também influencia a proliferação e a carcinogênese das glândulas mamárias caninas ${ }^{17}$. A exposição prolongada leva a hiperplasia de nódulos lobulares e adenomas simples ou complexos na glândula mamária. $O$ efeito proliferativo na glândula mamária coincide com o aumento das concentrações plasmáticas de HC. Tem-se observado que cães com concentrações endógenas elevadas de progesterona apresentam altas concentrações plasmáticas de HC.

\section{Tipos de tumores, classificação histopatológica e comportamento biológico.}

Aproximadamente $40 \%$ a $50 \%$ destes tumores mamários em cães são malignos. A classificação pode ser realizada de acordo com o tecido de origem (tecido epitelial, miogênico ou mesenquimatoso), características morfológicas descritivas e prognóstico. A classificação histológica internacional da Organização Mundial de Saúde dos tumores mamários do cão e do gato combina a classificação morfogênica histogênica e descritiva, incorporando características prognósticas histológicas associadas ao aumento da malignidade ${ }^{18}$. A maioria dos tumores da glândula mamária é de origem epitelial. Alguns, no entanto, podem ter uma histologia mista que consiste em tecido epitelial e mioepitelial, com áreas de cartilagem e osso, e alguns tumores 
Câncer da mama na cadela

Medeiros VB

são de origem puramente mesenquimatosa. Os tumores epiteliais são frequentemente classificados de acordo com as fronteiras histopatológicas e a diferenciação. Um carcinoma in situ é um tumor epitelial com características malignas que não invadiu a membrana basal. Essas lesões são muitas vezes multicêntricas e podem crescer em dutos ou lóbulos pré-existentes.

Os carcinomas ductais (adenocarcinomas) são o tipo mais comum de tumores das glândulas mamárias no cão. Os carcinomas sólidos são menos diferenciados. São tumores epiteliais indiferenciados, pleomórficos e infiltrativos que não são classificáveis em nenhuma das outras categorias de carcinomas $^{18}$. Os carcinomas inflamatórios da glândula mamária são carcinomas anaplásicos com características clínicas e histopatológicas características, como o envolvimento da pele subjacente com edema e dor, infiltrado celular inflamatório extenso, células epiteliais malignas nos linfáticos dérmicos e uma evolução clínica rápida, de mau prognóstico. A diferenciação histopatológica dos tumores das glândulas mamárias epiteliais tem impacto no prognóstico, com piora do prognóstico associado à perda de diferenciação. O carcinoma in situ e os adenocarcinomas apresentam o melhor prognóstico, e os carcinomas anaplásticos e inflamatórios apresentam o pior prognóstico ${ }^{18}$.

\section{Sinais clínicos e exame físico}

Os cães com tumores da glândula mamária são geralmente mais velhos, aproximadamente de 9 a 11 anos, sexualmente intactos ou esterilizados em idade mais $\operatorname{tardia}^{19}$. A maioria dos cães com tumores da glândula mamária são clinicamente saudáveis quando inicialmente apresentam-se para a avaliação de seus tumores. A duração dos sinais clínicos também varia muito de apenas alguns dias a muitos meses $^{20}$. Os tumores podem ter sido encontrados pelo proprietário ou podem ser um achado incidental durante o exame físico de rotina. Dependendo do tipo de tumor e de quanto tempo é detectado, os tumores podem ser pequenos, grandes, ulcerados, fixos, bem circunscritos ou envolvendo apenas uma ou várias glândulas. As glândulas mamárias 4 e 5 caudais são mais comumente envolvidas que as glândulas mais cranianas, mas a localização não parece afetar o prognóstico ${ }^{21}$. Não é incomum encontrar mais de um tumor em diferentes glândulas; mais de $60 \%$ dos casos têm mais 
Câncer da mama na cadela

Medeiros VB

de um tumor. Todos os tumores individuais devem ser biopsiados porque podem ser de tipos histopatológicos diferentes ${ }^{22}$. Tumores múltiplos não necessariamente implicam em pior prognóstico. O prognóstico é influenciado pelo tamanho, tipo e diferenciação dos tumores individuais. Os gânglios linfáticos regionais podem ser normais ou palpáveis, aumentados ${ }^{23}$.

Cães com doença metastática avançada ou carcinomas mamários inflamatórios normalmente apresentam sinais sistêmicos de doença quando são diagnosticados. Cães com doença metastática podem apresentar sinais inespecíficos como fadiga, letargia e perda de peso. A gravidade desses sinais depende da extensão e localização das metástases. Os cães com metástases podem ou não ter tumores óbvios das glândulas mamárias, dependendo se tiveram ressecção cirúrgica prévia de tumores primários. A maioria das metástases de tumor da glândula mamária ocorrem dentro de 1 ano da cirurgia inicial ${ }^{24}$.

Cães com carcinoma inflamatório de glândula mamária apresentam sinais clínicos mais intensos e graves. Sinais clínicos típicos incluem inflamação extensa das glândulas mamárias envolvidas com edema e dor. Esses cães, portanto, podem ser diagnosticados como com mastite. Muitas vezes, estão em condições clínicas precárias e têm fraqueza generalizada, perda de peso, poliúria e polidipsia e uma alta incidência de doença metastática, tanto para os linfonodos regionais como para os pulmões. 0 prognóstico é extremamente pobre com uma sobrevivência média de 25 dias após o diagnóstico ${ }^{25}$.

\section{Diagnóstico e estadiamento}

Os tumores mamários são o tumor mais comum em cães do sexo feminino ${ }^{26,27}$. Não têm predisposição por raça e afetam principalmente animais seni e aqueles não castrados na época certa. Vários sistemas foram propostos para avaliar o prognóstico desses animais. Um desses sistemas é o TNM, onde: T - descreve o tamanho do tumor; Metástase dos linfonodos N; e M - metástases à distância ${ }^{28}$. Existem estudos que mostram uma forte correlação entre o tamanho do tumor e malignidade ${ }^{29}$. Correlação entre a presença das metástases nos linfonodos e a sobrevivência do animal afetado ${ }^{30,31}$. Em pacientes do sexo feminino humano, o 
Câncer da mama na cadela

Medeiros VB

sistema TNM ainda é amplamente utilizado como fator preditivo para os carcinomas mamários, embora existam métodos mais modernos, como o dos biomarcadores ${ }^{32}$. Outro método que pode ser usado para a avaliação prognóstica de tumores é a classificação histológica. Os tumores de Grau III foram associados a um baixo tempo de sobrevivência ${ }^{33}$.

Uma biópsia cirúrgica, tipicamente uma biópsia excisional, é recomendada como a abordagem diagnóstica inicial de cães com tumores de glândula mamária. Esta biópsia fornecerá tecido para diagnóstico histopatológico e será terapêutica para cães com tumores benignos. Cães com tumores malignos pequenos e bem diferenciados podem ser curados por biópsia excisional se as margens de ressecção forem adequadas e bem respeitadas. Os aspirados com agulha fina nem sempre diferenciam com precisão os tumores epiteliais malignos e benignos. O estadiamento completo exige exames de sangue, incluindo hemograma completo, perfil de química sérica e análise de urina. Avaliação do tumor primário, incluindo tamanho, tipo e diferenciação histológica; avaliação dos linfonodos regionais; e radiografias torácicas em três incidências, geralmente completa a avaliação. O objetivo do estadiamento é avaliar a saúde geral e determinar a extensão do tumor. Além disso, o estadiamento também é necessário para o planejamento do tratamento.

O estado dos linfonodos regionais tem um forte impacto sobre a sobrevida em cães com tumores da glândula mamária ${ }^{23,34}$. Portanto, os linfonodos regionais devem ser avaliados em todos os cães com tumores malignos, de modo que o tratamento sistêmico pode ser iniciado em casos de metástase linfonodal regional. Os métodos para avaliar os linfonodos regionais incluem palpação, aspirado com agulha fina, biópsia Tru-cut ou extirpação dos linfonodos com as respectivas cápsulas.

Nunca é demais repetir, que todos os cães com tumores malignos das glândulas mamárias devem ter radiografias torácicas em três incidências. A radiografia ainda é o método de diagnóstico padrão para avaliação de pacientes veterinários para doença pulmonar metastática. A radiografia convencional pode detectar lesões pulmonares que variam de 6 a $8 \mathrm{~mm}$ de diâmetro. A capacidade de detectar metástases precoces pode ser melhorada usando tomografia computadorizada (TC) para metástases tão pequenas quanto $4 \mathrm{~mm}$ de diâmetro. A detecção precoce e o tratamento da doença metastática podem ter impacto na resposta e resultado em 
Câncer da mama na cadela

Medeiros VB

pacientes humanos e a TC tornou-se o método padrão de avaliação de pacientes com câncer humano para metástases pulmonares ${ }^{35}$. A ocorrência de doença metastática pulmonar sincrônica no momento do diagnóstico inicial tem sido de $35 \%$, podendo ser menor ${ }^{23}$.

A modernização na medicina veterinária, bem como o comprometimento do cliente e a conscientização sobre câncer em animais de estimação, podem ter levado a uma detecção precoce e, portanto, ao diagnóstico de cães com doença em estágio inicial. Os pulmões são o local mais comum para metástases à distância em cães com tumores malignos das glândulas mamárias, mas testes diagnósticos adicionais podem ser indicados e outros locais anatômicos podem ser avaliados de acordo com os sinais clínicos específicos de um paciente individual. As metástases para as vísceras abdominais ou para os linfonodos podem ocasionalmente ser observadas. Ultrassonografia abdominal ou radiografia podem ser úteis para detectar metástases abdominais.

\section{Tratamento}

As modalidades utilizadas no tratamento de mulheres com câncer de mama incluem cirurgia, radioterapia, terapia hormonal e quimioterapia, associadas ou não. A escolha da modalidade depende dos resultados de muitos grandes estudos prospectivos que avaliam a eficácia de vários tratamentos em grupos de pacientes estratificados de acordo com o estágio e fatores prognósticos bem estabelecidos. Em geral, os tratamentos se intensificam com o avançado estágio clínico e aumentando a gravidade dos fatores prognósticos.

Atualmente, a cirurgia é o tratamento mais aceito para cães com tumores da glândula mamária, e não há diretrizes bem estabelecidas para o tratamento além da cirurgia. A cirurgia é a base do tratamento para os tumores da glândula mamária canina e é a modalidade mais efetiva para o controle local do tumor. O tipo de cirurgia não parece influenciar a sobrevivência, desde que todo o tumor seja removido com margens histológicas livres de tumor ${ }^{36,37}$. A abordagem cirúrgica recomendada em cães com tumores da glândula mamária é realizar uma biópsia excisional do tumor. 0 tamanho da cirurgia, ou seja, a tumorectomia versus mastectomia simples, versus 
Câncer da mama na cadela

Medeiros VB

mastectomia radical, depende do tamanho do tumor primário. Uma biópsia excisional proporcionará tecidos para avaliação histopatológica e também pode fornecer controle local se as margens do tumor estiverem livres. A excisão cirúrgica pode ser curativa em cães com doença do estádio I e aqueles com carcinomas pequenos, não invasivos e bem diferenciados. Cães com tumores de alto grau ou maiores provavelmente desenvolverão doença metastática; no entanto, podem se beneficiar de terapia adicional ${ }^{38}$.

A mastectomia, seguida de terapia por irradiação, é frequentemente usada como alternativa à mastectomia radical, ou além da mastectomia radical em mulheres com grandes tumores primários ou mais de quatro linfonodos axilares positivos. Os estudos encontraram melhorias significativas no controle loco-regional e na sobrevivência específica do tumor em mulheres que receberam radioterapia pósmastectomia ${ }^{39,40}$. A terapia de radiação não foi ainda avaliada no tratamento de tumores de glândulas mamárias em cães, mas pode ser um adjuvante razoável para cirurgia em cães com sarcomas primários de glândula mamária porque esses tumores frequentemente se repetem localmente e em locais distantes.

O objetivo da terapia hormonal é prevenir a estimulação de estrogênio nas células de câncer de mama. Isso pode ser alcançado por várias estratégias diferentes, incluindo receptores de bloqueio com moduladores específicos de receptores de estrogênio, que são antagonistas de receptores, como o tamoxifeno. O tamoxifeno é atualmente o tratamento mais comumente prescrito para o câncer de mama em mulheres na pós-menopausa e é recomendado como o primeiro antiestrogênio.

Recomendações relativas ao tratamento adjuvante do câncer de mama precoce experimentou mudanças importantes nos últimos 25 anos em medicina humana. Desde meados da década de 1970, quando a ciclofosfamida, o metotrexato e o 5-fluorouracil resultaram em melhorias estatisticamente significativas e clinicamente significativas na sobrevivência livre e geral da doença, o uso de quimioterapia adjuvante tornou-se uma prática comum na terapia de câncer de mama humano ${ }^{41}$. As antraciclinas, como a doxorrubicina e a epirrubicina, têm sido consideradas entre os agentes mais ativos para tratar o câncer de mama e se tornaram um componente central dos regimes de adjuvantes na última década. Os regimes de quimioterapia adjuvante contendo uma antraciclina resultam em melhorias estatisticamente 
Câncer da mama na cadela

Medeiros VB

significativas em sobrevivência em comparação com regimes sem antraciclina ${ }^{42}$. A doxorrubicina mostrou-se eficaz em cães com metástase pulmonar e um relatório preliminar mostrou maior sobrevivência em cães com tumores de glândulas mamárias de alto risco que receberam doxorrubicina adjuvante em comparação com cães tratados com cirurgia isoladamente ${ }^{43}$.

\section{REFERÊNCIAS}

1. Liu D, Xiong H, Ellis AE, Northrup NC, Rodriguez CO Jr, O'Regan RM, et al. Molecular homology and difference between spontaneous canine mammary cancer and human breast cancer. Cancer Res. (2014) 74:5045-56.

2. Oakes SR, Gallego-Ortega D, Ormandy CJ. The mammary cellular hier-archy and breast cancer. Cell Mol Life Sci. 2014;71:4301-24.

3. Visvader JE, Stingl J. Mammary stem cells and the differentiation hierarchy: current status and perspectives. Genes Dev. 2014;28:1143-58.

4. Moe L. Population-based incidence of mammary tumors in some dog breeds. J Reprod Fertil-Suppl. 2001;57:439-43.

5. Stovring M, Moe L, Glattre E. A population based case-control study of canine mammary tumors and clinical use of medroxyprogesterone acetate. APMIS. 1997;105:590-6.

6. Selman PJ, Mol JA, Rutteman GR, et al. Progestin-induced growth hormone excess in the dog originates in the mammary gland. Endocrinology. 1994;134:287-92.

7. Hulka BS, Edison TL, Lininger RA. Steroid hormones and risk of breast cancer. Cancer Supp. 1994;74(3):1111-24.

8. Rangel MC, Bertolette D, Castro NP, Klauzinska M, Cuttitta F, Salomon DS. Developmental signaling pathways regulating mammary stem cells and contributing to the etiology of triplenegative breast cancer. Breast Cancer Res Treat. 2016;156:211-26.

9. Van Keymeulen A, Rocha AS, Ousset M, Beck B, Bouvencourt G, Rock J, et al. Distinct stem cells contribute to mammary gland development and maintenance. Nature 2011;479:189-93.

10. Chlebowski RT, Hendrix SL, Langer RD, Stefanick ML, Gass M, Lane D, et al. Influence of estrogen plus progestin on breast cancer and mammography in healthy postmenopausal women: the Women's Health Initiative randomized trial. JAMA 2003;289:3243-53.

11. Beatson GT. On the treatment of the inoperable cases of carcinoma of the mamma: suggestions for a new method of treatment with illustrative cases. Lancet 1896;2:104-7.

12. Huggins C, Yang NC. Induction and extinction of mammary cancer. A strik-ing effect of hydrocarbons permits analysis of mechanisms of causes and cure of breast cancer. Science 1962;137:257-62.

13. McGuire WL, Horwitz KB. A role for progesterone in breast cancer. Ann N Y Acad Sci. 1977;286:90-100.

14. MacMahon B, Cole $P$, Lin TM, Lowe CR, Mirra AP, Ravnihar B, et al. Age at first birth and breast cancer risk. Bull World Health Organ. 1970;43:209-21.

15. Swanson SM, Unterman TG. The growth hormone-deficient spontaneous Dwarf rat is resistant to chemically induced mammary carcinogenesis. Carcinogenesis 2002;23:977-82. 
Câncer da mama na cadela

Medeiros VB

16. Russo J, Russo IH. The role of estrogen in the initiation of breast cancer. J Steroid Biochem Mol Biol. 2006;102:89-96.

17. Misdorp W. Canine mammary tumours: protective effect of late ovariectomy and stimulating effect of progestins. Vet Q. 1988;10:26-33

18. Misdorp W, Else RW, Hellmen E, et al. Histological classification of mammary tumors of the dog and cat. WHO International Histological Classification of Tumors of Domestic Animals. 2nd Series, vol VII. Washington DC: Armed Forces Institute of Pathology, American Registry of Pathology; 2001.

19. Kurzman ID, Gilbertson SR. Prognostic factors in canine mammary tumors. Semin Vet Med Surg. 1986;1(1):25-32.

20. Perez Alenza MD, Tabanera E, Pena L. Inflammatory mammary carcinoma in dogs: 33 cases (1995-1999). J Am Vet Med Assoc. 2001;219(8):1110-4.

21. Hellemen E, Bergstrom R, Holmberg L. Prognostic factors in canine mammary gland tumors: a multivariate study of 202 consecutive cases. Vet Pathol. 1993;30:20-7.

22. Benjamin SA, Lee AC, Saunders WJ. Classification and behavior of canine mammary epithelial neoplasms based on life-span observations in beagles. Vet Pathol. 1999;36(5):42336.

23. Yamagami T, Kobayashi T, Takahashi K. Prognosis for canine malignant mammary tumors based on the TNM and histologic classification. J Vet Med Sci. 1996;58(7):1079-83.

24. Fidler IJ, Abt DA, Brody RS. The biological behavior of canine mammary neoplasms. J Am Vet Med Assoc. 1967;151(10):1311-8.

25. Perez Alenza MD, Tabanera E, Pena L. Inflammatory mammary carcinoma in dogs: 33 cases (1995-1999). J Am Vet Med Assoc. 2001;219(8):1110-4.

26. Cassali GD, Lavalle $G E$, de Nardi $A B$, et al. Consensus for the diagnosis, prognosis and treatment of canine mammary tumors. Braz J Vet Pathol. 2011;4:153-180.

27. Cassali GD. Comparative mammary oncology: Canine model. BMC Proceedings. 2013;7:110.

28. Owen LN. TNM Classification of Tumors in Domestic Animals,1st ed. Geneva, Switzerland: World Health Organization, 1980:1-53.

29. Sorenmo KU, Kristiansen VM, Cofone MA, et al. Canine mammary gland tumours; a histological continuum from benign to malignant; clinical and histopathological evidence. Vet Comp Oncol. 2009;7:162-172.

30. de Araujo MR, Campos LC, Ferreira E, Cassali GD. Quantitation of the regional lymph node metastatic burden and prognosis in malignant mammary tumors of dogs. J Vet Intern Med. 2015;29:1360-1367.

31. Dong $G$, Wang $D$, Liang $X$, et al. Factors related to survival rates for breast cancer patients. Int J Clin Exp Med. 2014;7:3719-3724.

32. Orucevic A, Chen J, McLoughlin JM, Heidel RE, Panella T, Bell J. Is the TNM staging system for breast cancer still relevant in the era of biomarkers and mmerging personalized medicine for breast cancer - An institution's 10-year experience. Breast J. 2015;21:147-154.

33. Karayannopoulou M, Kaldrymidou E, Constantinidis TC, Dessiris A. Histological grading and prognosis in dogs with mammary carcinomas: Application of a human grading method. J Comp Path. 2005;133:246-252.

34. Yamagami T, Kobayashi T, Takahashi K. Prognosis for canine malignant mammary tumors based on the TNM and histologic classification. J Vet Med Sci. 1996;58(7):1079-83. 
Câncer da mama na cadela

Medeiros VB

35. Hellemen E, Bergstrom R, Holmberg L. Prognostic factors in canine mammary gland tumors: a multivariate study of 202 consecutive cases. Vet Pathol. 1993;30:20-7.

36. Glasspool RM, Evans TRJ. Clinical imaging of cancer metastasis. Eur J Cancer. 2000;36(13):1661-70.

37. Allen SW, Mahaffey EA. Canine mammary neoplasia: prognostic indicators and response to surgical therapy. J Am Anim Hosp Assoc. 1989;25:540-6.

38. MacEwen EG, Harvey HJ, Patnaik AK, et al. Evaluation of the effect of levamizole and surgery on canine mammary cancer. J Biol Resp Mod. 1985;4:418-26.

39. Kurzman ID, Gilbertson SR. Prognostic factors in canine mammary tumors. Semin Vet Med Surg. 1986;1(1):25-32.

40. National Institutes of Health Consensus Development Panel. National Institutes of Health Consensus Statement: adjuvant therapy for breast cancer. J Natl Cancer Inst Monogr. 2001;30:5-15.

41. Whelan TJ, Julian J, Wright J, et al. Does loco-regional radiation therapy improve survival in breast cancer? A meta-analysis. J Clin Oncol. 2000;18:1220-9.

42. Hortobagyi GN. Progress in systemic chemotherapy of primary breast cancer: an overview. J Natl Cancer Inst Monogr. 2001;30:72-9.

43. Piccart MJ, Lohrisch C, Duchateau L, et al. Taxanes in the adjuvant treatment of breast cancer: why not yet? J Natl Cancer Inst Monogr. 2001;30:88-95. 